\title{
Hydrodynamical interpretation of basic nebular structures
}

\author{
M. Steffen ${ }^{1}$ and D. Schönberner ${ }^{1}$ \\ ${ }^{1}$ Astrophysikalisches Institut Potsdam, 14482 Potsdam, Germany \\ email: msteffen@aip.de, deschoenberner@aip.de
}

\begin{abstract}
Based on existing hydrodynamical simulations, we review our present understanding of the formation and evolution of planetary nebulae $(\mathrm{PNe})$ and discuss the relevant processes responsible for the development of the basic (1D) nebular structures.
\end{abstract}

Keywords. hydrodynamics - radiative transfer - stars: evolution - stars: mass loss - planetary nebulae: general, individual (IC 418, NGC 6826, NGC 2438, Abell 39, NGC 7027, NGC 6543)

\section{Hydrodynamical modeling of planetary nebulae}

In this review we concentrate on the basic structures of planetary nebulae, i.e. those spherical (1D) structures which must inevitably arise as a consequence of the final evolution of a single, non-rotating, non-magnetic star of intermediate mass. These basic structures will still be relevant if there are some deviations from spherical symmetry, like for elliptical PNe. Since round+elliptical PNe make up the largest fraction of planetary nebulae (e.g. Manchado 2003), a sound physical understanding of their basic structures is essential.

The ideal way to understand the structure and evolution of planetary nebulae is to start from theoretical concepts and to develop corresponding models including the essential physics. This procedure can be considered successful if the model nebulae arising in the computer can be identified with observed objects. As pointed out by B. Gustafsson (this volume), this approach is clearly more satisfactory and instructive than the reverse empirical approach where one builds models with a number of free parameters which are adjusted until the model fits certain observed features. The essential physical ingredients for the modeling of planetary nebulae are (i) AGB and post-AGB stellar evolution with mass loss, providing the time evolution of the stellar radiation field and the wind properties; (ii) radiation hydrodynamics of stellar winds, describing the dust-driven winds on the AGB, and the wind-wind interaction during the post-AGB evolution; (iii) radiative transfer and non-equilibrium low-density plasma physics for a proper treatment of time-dependent photo-ionization/recombination at densities where the atomic recombination time scales are comparable or even larger than the time scales of the rather rapid evolution of the central star and the dynamical evolution of the nebula. All these ingredients have been incorporated into the 'Potsdam NEBEL code', as described by Marten \& Szczerba (1997), Perinotto et al. (1998, 2004) and references therein.

A similar approach of modeling spherical PNe has been adopted by Villaver et al. (2002a,b): they account for stellar evolution with mass loss, and study the dynamical evolution of circumstellar gas envelopes around AGB and post-AGB stars using the ZEUS-3D code. However, radiation transfer is not included in this code, and ionization is treated in the Strömgren approximation, assuming instantaneous ionization equilibrium. Within the ionized region, the (electron) temperature is set to a fixed value of $10000 \mathrm{~K}$, independent of the effective temperature of the central star. Since the ionization 
stratification of individual ions cannot be described in the framework of this model, the calculation of synthetic metal line profiles is not possible either. In its $2 \mathrm{D}$ configuration, the code has been used to simulate the interaction of PNe with the interstellar medium (Villaver et al. 2003). The 2D models by Mellema (1994, 1995, 1997) include all the relevant physics, except that the metal ions are treated in equilibrium. Since the physical origin of axisymmetric PNe is yet unknown, additional free parameters are introduced to describe the aspherical density distribution of the AGB wind. An updated version of this code, including a fully time-dependent treatment of ionization and recombination, was recently employed for 1D simulations of the ring system in the inner halo of NGC 6543 (Hyung et al. 2001; Meijerink et al. 2003). Since the Potsdam group provided the most recent and most comprehensive grid of hydrodynamical PN models, incorporating the most detailed physics, we discuss in the following Sections the formation and evolution of the basic structures of round/elliptical planetary nebulae mainly based on their current $1 \mathrm{D}$ models. The discussion of more complex, non-spherical structures, presumably generated by stellar rotation, magnetic fields, or binarity requires $2 \mathrm{D}$ or $3 \mathrm{D}$ simulations and is beyond the scope of this review.

\section{Formation and evolution of PNe: models and observations}

\subsection{Final evolution on the AGB, formation of a proto planetary nebula}

A proper modeling of planetary nebulae starts already on the upper AGB, since the environment out of which they form is set up by the preceding mass loss history. The dynamical evolution of the dust-driven outflow from cool giant stars during their final few 100000 years on the AGB has been computed by Steffen et al. (1998) using a two-component (gas/dust) radiation-hydrodynamics code (see also Steffen \& Schönberner 2000), coupled to stellar evolution calculations with mass loss according to Blöcker (1995a). Simulations are available for tracks of different initial/final stellar mass, assuming both oxygenand carbon-rich dust composition. The considerable luminosity variation over a thermal pulse cycle $(P \approx 50000 \ldots 100000 \mathrm{yr})$ leads to a corresponding modulation of the mass loss rate. As a consequence, the circumstellar wind envelope of AGB stars exhibits periodic minima in the radial density structure, reflecting the mass loss history over several thermal pulse cycles. Typical outflow velocities are $10 \ldots 15 \mathrm{~km} / \mathrm{s}$.

The radial profiles of gas density and outflow velocity obtained from these detailed simulations at the end of the AGB $(t=0)$ have been adopted as initial conditions for computing the evolutionary sequences of hydrodynamical PN models referred to in the following (for details see Perinotto et al. 2004). It is important to note that the predicted density profile at the end of the AGB can be approximated by the relation $\rho \sim r^{-\alpha}$, with $\alpha \approx 3$ (cf. Steffen et al. 1998, Fig. 19). This behavior reflects the steadily increasing Blöcker (1995a) mass loss rate towards the end of the AGB. In contrast, Villaver et al. (2002a) report that $\alpha \approx 2$ gives a reasonable fit to the density structure of their AGB wind envelopes. Presumably, this is related to the truncation of the mass loss rate in the adopted parameterization by Vassiliadis \& Wood (1993). Interestingly, a recent study of the observed $\mathrm{CO}$ emission line profiles of a sample of proto planetary nebulae (PPNe) by Hrivnak \& Bieging (2005) concludes that the density law in the circumstellar envelopes of these early post-AGB objects cannot be fitted by $\alpha \approx 2$, but rather requires $\alpha \approx 3 \ldots 4$. A similar result $(\alpha>2.5)$ has been obtained by Kwok et al. (2002) from model fits to the observed IR brightness profiles of two selected PPNe.

\subsection{The first 3000 years of post-AGB evolution}

After the star has evolved off the AGB due to the heavy mass loss, the increasing ionizing photon flux and dynamical impact of the fast wind of the hot remnant quickly reshape 

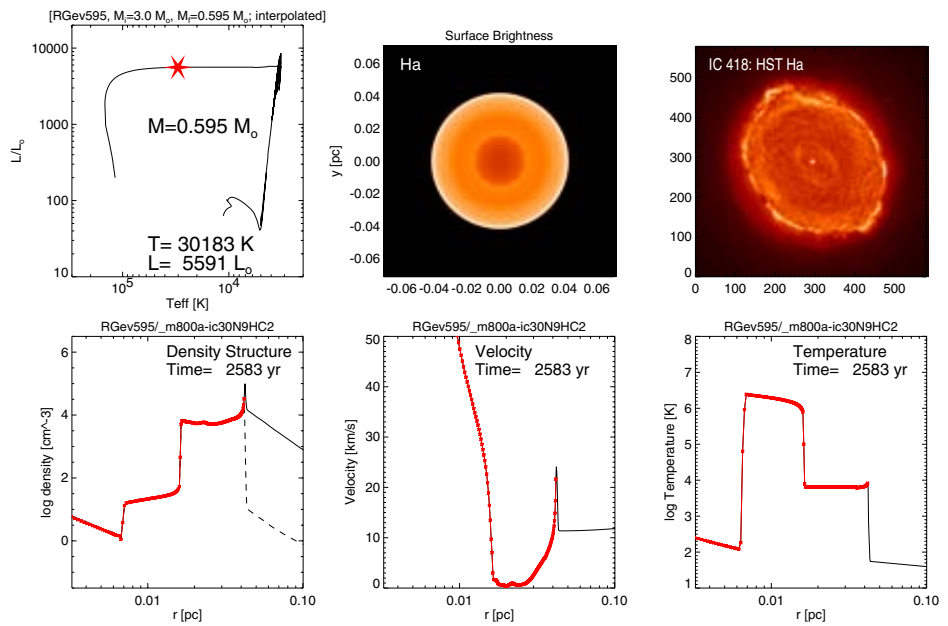

Figure 1. Snapshot from a model sequence with a central star of $0.595 \mathrm{M}_{\odot}$, after 2600 years of post-AGB evolution. Top left to bottom right: position of the central star in the physical Hertzsprung-Russell diagram; $\mathrm{H} \alpha$ surface brightness of the corresponding model PN; HST H $\alpha$ image of $I C 418$; heavy-particle (solid) and electron (dashed) density of model PN; expansion velocity; electron temperature. Regions where hydrogen is more than $90 \%$ ionized are drawn as thick (red) lines (lower panels). Heat conduction is taken into account, reducing $T$ (hot bubble).

the inner parts of the circumstellar AGB envelope into a planetary nebula. The structure of a planetary nebula has therefore no direct relation to the previous mass loss history.

We follow the evolution of a central star (CS) of $M_{\mathrm{CS}}=0.595 \mathrm{M}_{\odot}$ (Blöcker 1995b, interpolated). According to dedicated radiation hydrodynamics simulations (cf. Perinotto et al. 2004), the sequence of events is as follows. First, the circumstellar matter is everywhere neutral, and temperatures do not exceed a few $100 \mathrm{~K}$. After $\approx 750 \mathrm{yr}$, a thin layer of compressed gas has formed at the interface between the fast CS wind $\left(V_{\mathrm{CS}} \approx 75 \mathrm{~km} / \mathrm{s}\right)$ and the slow $\mathrm{AGB}$ wind $\left(V_{\mathrm{AGB}} \approx 12 \mathrm{~km} / \mathrm{s}\right)$, with peak temperatures of $T_{\text {interface }} \approx 10000 \mathrm{~K}$. After $1000 \mathrm{yr}$, the stellar effective temperature has reached $T_{\text {eff }} \approx 10000 \mathrm{~K}$, and the fast wind as well as the compressed layer are now fully ionized and heated up by ionization $\left(T_{\text {wind }} \approx 1000 \mathrm{~K}, T_{\text {interface }} \approx 10^{5} \mathrm{~K}\right)$. During the next 500 years, $T_{\text {eff }}$ rises to $\approx 18000 \mathrm{~K}$ and an ionized shell with $T_{\text {shell }} \approx 10000 \mathrm{~K}$ forms at the leading edge of the interface, bounded by a D-type ionization front. At the same time, the velocity of the fast wind increases to $V_{\mathrm{CS}} \approx 300 \mathrm{~km} / \mathrm{s}$ and develops an inner shock where the kinetic energy of the wind is dissipated into heat, leading to the formation of a hot bubble with $T_{\text {bubble }}>10^{6} \mathrm{~K}$ immediately behind the leading ionized shell. The basic structures have thus emerged after only 1500 years of post-AGB evolution.

Figure 1 shows the situation another 1000 years later. At that time $\left(T_{\text {eff }} \approx 30000 \mathrm{~K}\right)$, the appearance of the model closely resembles that of the young planetary nebula $I C$ 418 (Fig. 1, top right). Identification of model and observation suggests that $I C 418$ is still optically thick in the Lyman continuum, and that we see essentially the young shell which is driven by photo-ionization. Its sharp outer edge is the signature of a D-type ionization front. In both model and observation the future 'rim' is already visible, but its faintness indicates that the impact of the CS wind is still weak. It is worth mentioning that not only the surface brightness structure but also the kinematics of model and observation are in general agreement: while the central line profiles of [O III] are unsplit, indicating very low expansion velocities, the Doppler splitting of the [N II] lines indicates an expansion velocity of $V_{\exp } \approx 15 \mathrm{~km} / \mathrm{s}$. This result can be explained by the positive 

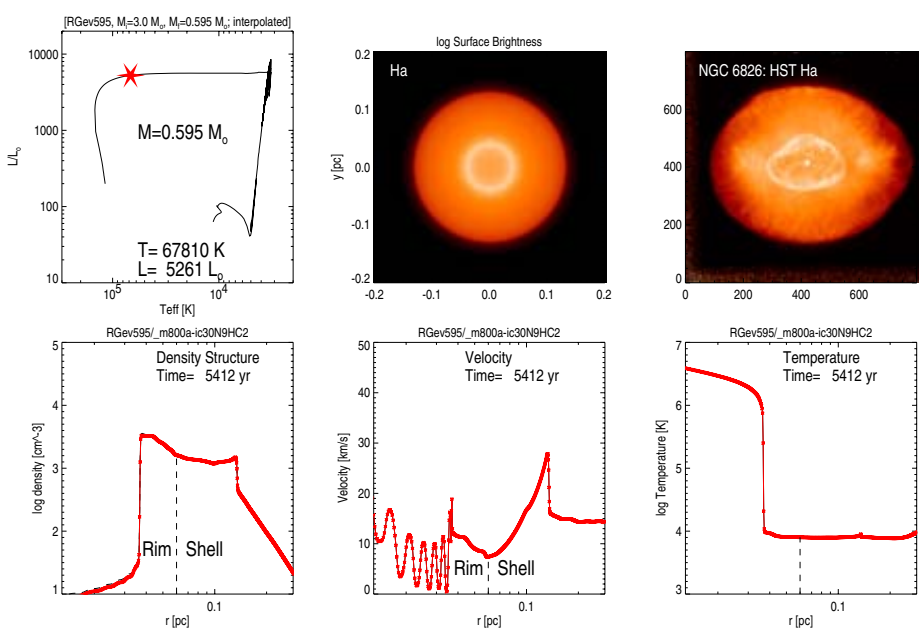

Figure 2. Same as Fig. 1, but at a later time, $t=5400 \mathrm{yr}$. This fully ionized configuration represents typical double-shell PNe like NGC 6826 (upper left, HST $\mathrm{H} \alpha$ image courtesy B. Balick). The vertical dashed line in the lower panels marks the interface between rim and shell.

velocity gradient in the shell (Fig. 1, bottom center) in combination with the different ionization stratifications of $\mathrm{N}$ and $\mathrm{O}$.

\subsection{The formation of a double-shell planetary nebula}

As time proceeds, the ionization-driven shell expansion accelerates (cf. Schönberner et al. $2005 \mathrm{a}, \mathrm{b})$ as the central star becomes hotter. The gas velocity immediately behind the Dtype ionization front increases from $V_{\text {post }} \approx 23 \mathrm{~km} / \mathrm{s}$ to $\approx 30 \mathrm{~km} / \mathrm{s}$ between $t=2500$ and $4000 \mathrm{yr}$. At that time, the increased number of ionizing photons $\left(T_{\mathrm{eff}} \approx 48000 \mathrm{~K}\right)$ and the decreasing gas density $\left(n \approx 10^{4} \mathrm{~cm}^{-3}\right)$ finally allow the ionization front to break free. As the front races outwards, it heats up the surrounding cool AGB matter to temperatures initially in excess of $20000 \mathrm{~K}$ (cf. Sandin et al., this volume). The nebula is now optically thin, and density bounded, surrounded by a faint extended halo which still preserves a record of the heavy mass-loss at the end of the AGB.

During the optically thick phase, the maximum density is found at the outer edge of the shell. After the passage of the ionization front, the outer density peak weakens. At the same time, the density at the inner edge of the nebula increases (relative to the density at the outer edge) due to the increasing pressure exerted by the central hot bubble, as the wind power, $L_{\text {wind }}=\dot{M}_{\mathrm{CS}} V_{\mathrm{CS}}^{2} / 2$, grows steadily from $0.6 \mathrm{~L}_{\odot}$ at $t=2500 \mathrm{yr}$ to $2.6 \mathrm{~L}_{\odot}$ at $t=5000 \mathrm{yr}$. Now the maximum density is found at the inner edge of the nebula, representing what is observationally known as the rim, surrounded by a shell of distinctly lower density. A double-shell nebula has formed.

The structure that has emerged after 5400 years of post-AGB evolution is shown in Fig. 2. It is representative of a typical double-shell nebula like e.g. NGC 6826. A more detailed comparison of observed and synthetic surface brightness profiles in different emission lines for a selected sample of double-shell planetary nebulae confirms the validity of the hydrodynamical models (see Schönberner et al. 2005b, Fig.7).

The kinematics can be studied by means of high-resolution emission line profiles. As demonstrated in Fig. 3, both observation and model indicate distinctly different expansion velocities for rim and shell: the Doppler splitting of the main component reflects the average expansion of the $\operatorname{rim}\left(V_{\text {rim }} \approx 8 \mathrm{~km} / \mathrm{s}\right.$ ), while the weak 'shoulders' (first noted by Chu et al. 1984) are due to the faster expansion of the shell $\left(V_{\text {shell }} \approx 24 \mathrm{~km} / \mathrm{s}\right)$. This 

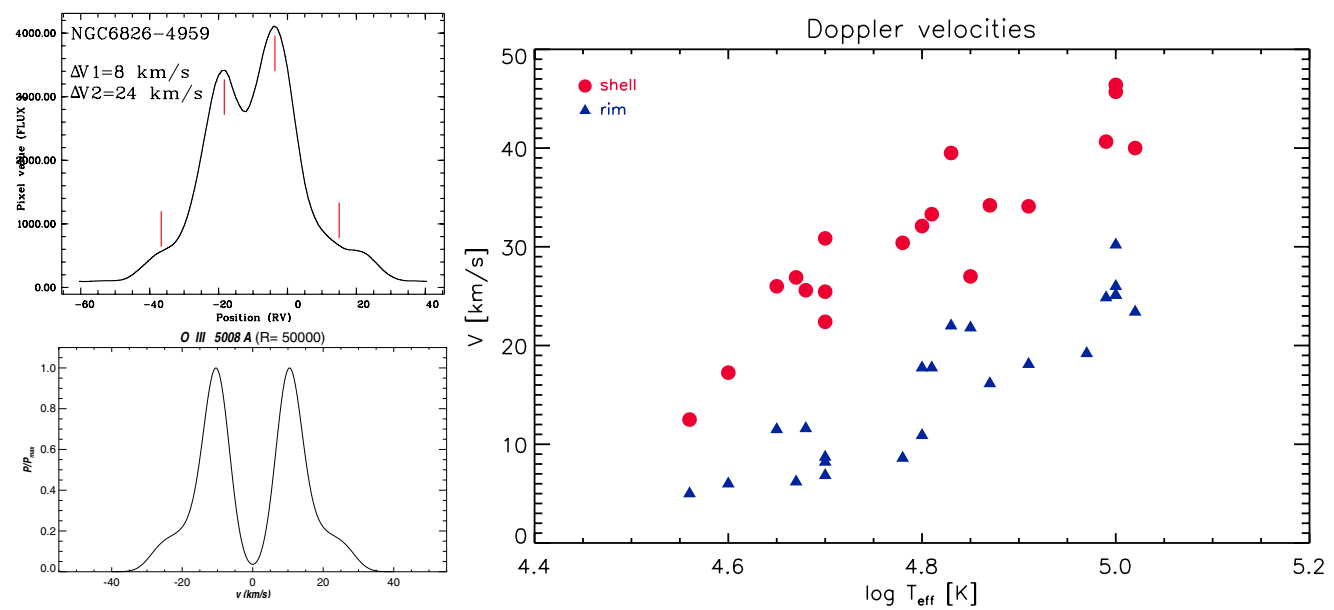

Figure 3. Observed central line profile of [O III], $\lambda 5007 \AA$ of $N G C 6826$ (top left, unpublished data from Lehmann \& Hildebrandt) compared with the corresponding line computed from the model shown in Fig. 2, assuming a spectral resolving power of $R=50000$ (bottom left). Right: shell (circles) and rim (triangles) expansion velocities of a sample of selected double-shell $\mathrm{PNe}$ plotted as a function the effective temperature of the respective central stars, as obtained from a Gaussian decomposition of the central [O III] Doppler profiles (source: Schönberner et al. 2005a plus Lehmann \& Hildebrandt).

behavior is in fact characteristic for double-shell nebulae. $V_{\text {shell }}$ is generally larger than $V_{\text {rim }}$, and both velocities increase with effective temperature or time (Fig. 3, right panel).

The hydrodynamical models tell us that rim and shell expand independently since they are driven by different causes: the expansion of the rim depends on the strength of the CS wind and the ambient shell density (thus indirectly on $\dot{M}_{\mathrm{AGB}}$ ), whereas the expansion of the shell is determined primarily by the gradient of the ambient AGB wind (and to a lesser extent by the sound speed, i.e. gas temperature). The models further teach us that the kinematic age derived from the Doppler splitting of the central line profiles and the radius of the rim is expected to be almost independent of the real age of the $\mathrm{PN}$, and hence gives spurious results. In contrast, combining the [N II] Doppler velocity of the shell with its radius gives a close approximation to the true PN age (for details see Schönberner et al. 2005b). The hydrodynamical models have also been utilized to compute correction factors for a proper evaluation of expansion parallaxes, compensating for the systematic difference between shock speed and Doppler velocities (cf. Mellema 2004), which has so far been ignored (e.g. Palen et al. 2002). For rim measurements, the correction factor can exceed 3 for young objects, while it is always less than 1.5 for the shell (Schönberner et al. 2005b). It should be noted at this point that the hydrodynamical simulations not only provide a convincing model of the density/ionization structure and the kinematics of double-shell PNe, but are also able to explain the faint haloes (Corradi et al. 2003) and the diffuse X-ray emission of these nebulae (cf. Schönberner, Steffen \& Warmuth, this volume).

\subsection{Evolved planetary nebulae}

During the further evolution, the model PN expands as a double-shell configuration. Since the expansion is ruled by hydrodynamics, a ballistic expansion law is not a valid approximation (see Schönberner et al. 2005b, Fig. 5). As the luminosity of the central star drops below $L \approx 400 \mathrm{~L}_{\odot}$, the shell undergoes partial recombination, while rim and halo remain fully ionized. For this model $\left(M_{\mathrm{CS}}=0.595 \mathrm{M}_{\odot}\right)$, the recombination phase is 

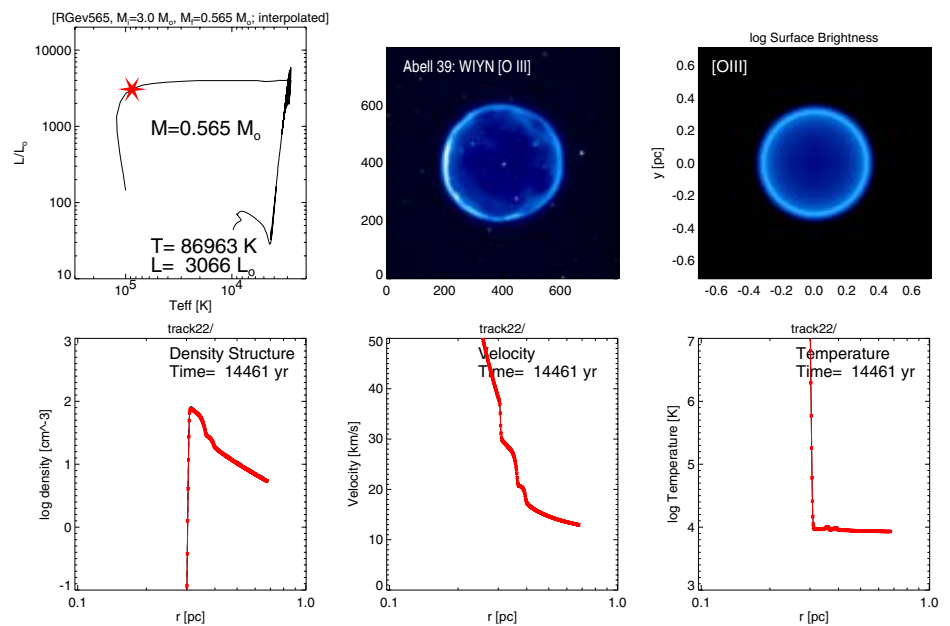

Figure 4. Snapshot from a model sequence with a central star of $0.565 \mathrm{M}_{\odot}$, after 14500 years of post-AGB evolution. The top center panel shows a WIYN [O III] image of Abell 39 (Jacoby et al. 2001), compared to the corresponding synthetic surface brightness map computed from the model (top right). Other panels as in Figs. 1 and 2.

rather short. After only 3000 years, the shell is fully re-ionized. In the case of a slightly more massive CS $\left(M_{\mathrm{CS}}=0.605 \mathrm{M}_{\odot}\right)$, recombination begins already when $L \approx 700 \mathrm{~L}_{\odot}$ and lasts for more than $6000 \mathrm{yr}$. A model from this sequence has been identified with the triple-shell planetary nebula NGC 2438 (Corradi et al. 2000). According to this interpretation, the bright inner halo of $N G C 2438$ is actually the recombined shell, while the faint outer halo represents the real halo, i.e. the fossil AGB wind. Nebulae with less massive central stars do not undergo a phase of recombination, they remain fully ionized. A late snapshot from a model sequence with a central star of $M_{\mathrm{CS}}=0.565 \mathrm{M}_{\odot}$ is shown in Fig. 4. This model has evolved from a double-shell to a single-shell configuration: since the rim finally exceeded the expansion rate of the shell, the latter was eventually 'swallowed' by the rim. This scenario can explain the appearance of the evolved spherical PN Abell 39. The synthetic [O III] surface brightness profile closely matches the observation, including the faint halo (not visible in Fig. 4). Hence, the hydrodynamical interpretation of the structure of Abell 39 is that it lost its shell because it was overrun by the rim. Abell 15 and Abell 20 are examples of slightly less evolved objects where part of the shell is still visible (see Emprechtinger et al. 2005). At the other extreme, nebulae with massive central stars never become optically thin in the Lyman continuum, because the stellar evolution is too rapid compared to the dynamical expansion of the circumstellar envelope. Nevertheless, such nebulae can develop a kind of double-shell structure according to the hydrodynamical models. NGC 7027 is probably an example of such an object, as discussed in Schönberner et al. (2005b).

\section{The influence of AGB mass loss history and central star wind}

Hydrodynamical test calculations have shown that the expansion properties and morphologies of the model PNe depend to some extend on the initial density structure of the surrounding circumstellar AGB matter. In this sense, planetary nebulae can in fact provide constraints on the final mass loss episode on the AGB. Figure 5 shows the striking result of a simple experiment: adopting a $\rho \sim r^{-2}$ law for the density distribution of the circumstellar envelope at the end of the AGB evolution instead of the results 

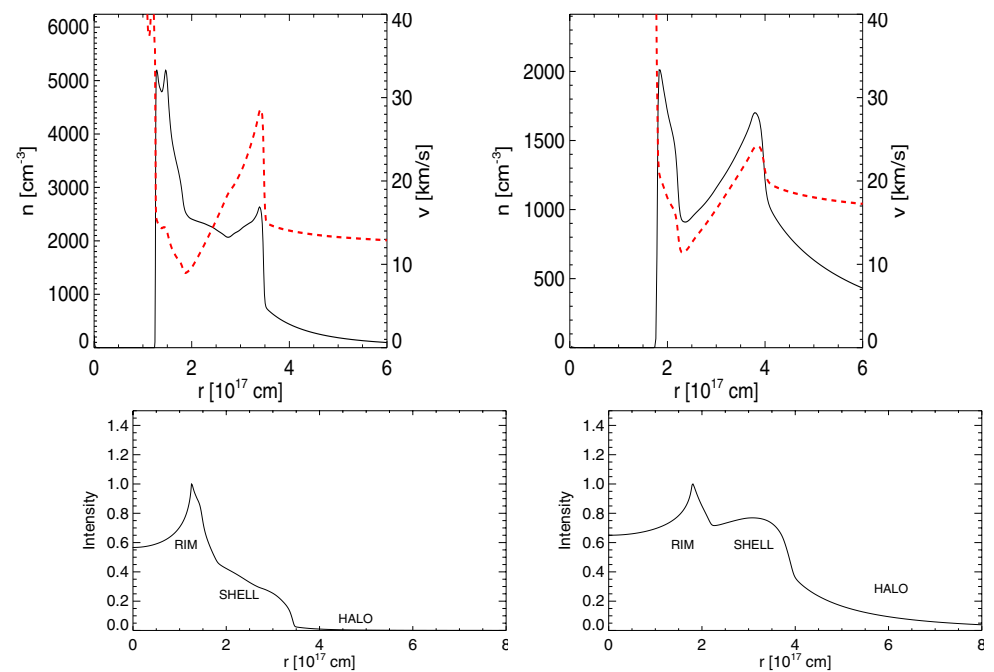

Figure 5. Radial profile of heavy-particle density (solid, top) and velocity (dashed, top), and $\mathrm{H} \alpha$ surface brightness distribution (bottom) for two models which differ in the radial density law of the initial circumstellar envelope: $\rho$ from hydrodynamical AGB wind model (left column), and assuming $\rho \sim r^{-2}$ (right column). The otherwise identical sequences both have a central star of $0.605 \mathrm{M}_{\odot}$ and are compared at time $t \approx 4400 \mathrm{yr}\left(T_{\mathrm{eff}} \approx 83000 \mathrm{~K}, L \approx 5700 \mathrm{~L}_{\odot}\right)$.
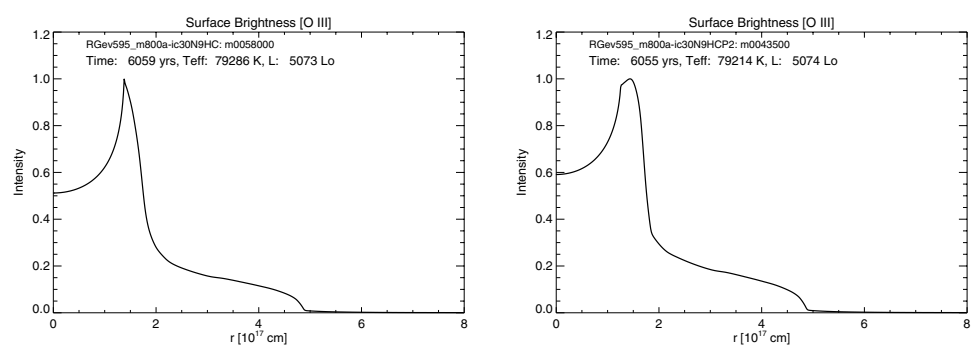

Figure 6. [O III] surface brightness profiles of two models which differ in the formulation of the central star wind: standard wind model (Pauldrach et al. 1998, left), and revised wind model (Pauldrach et al. 2004, right). The otherwise identical sequences both have a central star of $0.595 \mathrm{M}_{\odot}$ and are compared at time $t \approx 6050 \mathrm{yr}\left(T_{\text {eff }} \approx 80000 \mathrm{~K}, L \approx 5100 \mathrm{~L}_{\odot}\right)$.

from detailed hydrodynamical AGB wind calculations (where $\rho \sim r^{-\alpha}, \alpha \approx 3$ ), leads to a much enhanced density and a reduced expansion velocity of the shell. The resulting surface brightness profile becomes highly unrealistic, with a shape that is unlike any observed brightness distribution. In particular, the brightness ratio rim/shell and rim/halo is clearly too low. Likewise, the expansion velocity of the shell (which depends primarily on the slope $\alpha$ ) is distinctly lower for the $\alpha=2$ test model than observed. This experiment thus provides independent evidence for an increasing mass loss at the end of the AGB, with $\alpha \approx 3$ (cf. Section 2.1).

Concerning the influence of the central star wind on the appearance of the nebula, we have compared two sequences with different prescriptions of the CS mass loss rate. In the standard formulation based on Pauldrach et al. (1998), the wind power grows monotonically as a function of post-AGB age $\left(T_{\text {eff }}\right)$, while it passes through a maximum near $T_{\text {eff }} \approx 50000 \mathrm{~K}$ in the revised formulation by Pauldrach et al. (2004). This results in different properties of the rim for models evolved beyond the time of maximum wind power: for simulations employing the new wind model, the rim is geometrically thicker 
than in the standard models, the maximum density is now at the outer edge of the rim, and its velocity gradient is positive (like in the shell). A comparison of the surface brightness profiles is shown in Fig. 6. Observed rim properties (Sabbadin 2006, priv. comm.) seem to favor the revised CS wind theory.

\section{Conclusions}

Time-dependent 1D simulations combining stellar evolution with mass loss, radiationhydrodynamics of stellar winds, and non-equilibrium low-density plasma physics have proven to be an effective approach to explain the observed basic structures of planetary nebulae. The resulting hydrodynamical models can consistently describe (a) the X-ray emission from the central hot bubble, (b) the radial intensity profiles in various emission lines of different elements and ionization stages, (c) the internal kinematics and expansion properties, and $(\mathrm{d})$ the structure and evolution of the faint AGB haloes. This is a remarkable success. Based on the basic physical mechanisms governing the evolution of planetary nebulae, the hydrodynamical models allow a classification of PNe in terms of evolutionary state and central star mass. The largest uncertainties in PN modeling arise from the poor knowledge of the mass loss laws on the final AGB and beyond, and from the related ignorance of the so called transition times.

\section{References}

Blöcker, T. 1995a, A\&\&A, 297, 727

Blöcker, T. 1995b, A\& A, 299, 755

Corradi, R.L.M., Schönberner, D., Steffen, M., \& Perinotto, M. 2000, A\&\&A, 453, 1071

Corradi, R.L.M., Steffen, M., Schönberner, D., \& Perinotto, M. 2003, in: Planetary Nebulae: Their Evolution and Role in the Universe, IAU Symposium 209, 455

Chu, Y.-H., Kwitter, K.B., Kaler, J.B., \& Jacoby, G.H. 1984, PASP, 96, 598

Emprechtinger, M., Rauch, T., \& Kimeswenger, S. 2005, A\&A, 431, 215

Hrivnak, B.J. \& Bieging, J.H. 2005, ApJ, 624, 331

Hyung, S., Mellema, G., Lee, S.-J., \& Kim, H. 2001, A\&\&A, 378, 587

Jacoby, G.H., Ferland, G.J., \& Korista, K.T. 2001, ApJ, 560, 272

Kwok, S., Volk, K., \& Hrivnak, B.J. 2001, ApJ, 573, 720

Manchado, A. 2003, in: S. Kwok, M. Dopita, \& R. Sutherland (eds.), Planetary Nebulae: Their Evolution and Role in the Universe, IAU Symposium 209, 431

Marten, H. \& Szczerba, R. 1997, A\&A, 325, 1132

Meijerink, R., Mellema, G., \& Simis, Y. 2003, A\& $A$, 405, 1075

Mellema, G. 1994, A\&A, 290, 915

Mellema, G. 1995, MNRAS, 277, 173

Mellema, G. 1997, A\&A, 321, L29

Mellema, G. 2004, A\&A A, 416, 623

Palen, S., Balick, B., Hajian, A.R., Terzian, Y., Bond, H.E., \& Panagia, N. 2002, AJ, 123, 2666

Pauldrach, A., Puls, J., Kudritzki, R.P., Méndez, R.H., \& Heap, S.H. 1998, A\&GA 207, 123

Pauldrach, A., Hoffmann, T.L., \& Méndez, R.H. 2004, A\& A 419, 1111

Perinotto, M., Kifonidis, K., Schönberner, D., \& Marten, H. 1998, A\&A, 332, 1044

Perinotto, M., Schönberner, D., Steffen, M., \& Calonaci, C. 2004, A\& A, 414, 993

Schönberner, D., Jacob, R., Steffen, M., Perinotto, et al. 2005a, A\&SA, 431, 963

Schönberner, D., Jacob, R., \& Steffen, M. 2005b, A\&AA, 441, 573

Steffen, M., Szczerba, R., \& Schönberner, D. 1998, A\&A, 337, 149

Steffen, M. \& Schönberner, D. 2000, A\& $A, 357,180$

Vassiliadis, E. \& Wood, P. 1993, ApJ, 413, 641

Villaver, E., Garcia-Segura, G., \& Manchado, A. 2002a, ApJ, 571, 880

Villaver, E., Manchado, A., \& Garcia-Segura, G. 2002b, ApJ, 581, 1204

Villaver, E., Garcia-Segura, G., \& Manchado, A. 2003, ApJ, 585, L49 